\title{
Title: Brain-enriched CACNA1C isoforms as novel, selective targets for psychiatric indications
}

Nicola AL Hall ${ }^{1,2}$ and Elizabeth M Tunbridge ${ }^{1,2}$

1. Department of Psychiatry, University of Oxford, Oxford, UK

2. Oxford Health NHS Foundation Trust, Oxford, UK

Multiple lines of evidence suggest the Cav1.2 voltage-gated calcium channel (VGCC), encoded by CACNA1C, as a novel therapeutic target for psychiatric disorders. CACNA1C shows robust transdiagnostic associations with psychiatric disorders [1]. Cav1.2 is 'druggable' via the existing calcium channel blockers that are prescribed for cardiovascular indications but show evidence of psychiatric benefit [1]. It has a well-described biology and plays a role in neurobiological processes relevant to psychiatric disorders, including synaptic plasticity, and learning and memory [1].

There is a need to identify more selective Cav1.2 targets; repurposing the existing calcium channel blockers is unlikely to be feasible due to their cardiovascular effects, which represent side effects in the context of psychiatric conditions. CACNA1C encodes a family of Cav1.2 isoforms via alternative splicing mechanisms that differ between tissues to produce functionally-distinct Cav1.2 channels that vary in many parameters, including biophysical properties [2] and differing sensitivity to existing calcium channel blockers [3], highlighting that whilst challenging it may be feasible to develop isoform-selective compounds. Thus, brain-enriched isoforms of CACNA1C/Cav1.2 may represent selective therapeutic targets for psychiatry. However, the identification of such targets was hampered by two key limitations: firstly, most information about CACNA1C isoform diversity was obtained in rodents and splicing patterns can differ markedly between species [4]; secondly, because annotated transcripts are typically reconstructed computationally from short-read sequencing data, little was known about the full-length architecture of CACNA1C isoforms in any species. Therefore, we developed a novel technical approach to characterise full length isoforms and obtained the isoform profile of CACNA1C in human brain [5]. We identified 241 novel CACNA1C isoforms, although most were 
expressed at low levels [5]. However, we obtained our data using bulk tissue, so isoforms expressed at low levels in the pool could be abundant in specific cellular subpopulations. We are now using this method to profile the isoform repertoire of the human cardiovascular system. We believe it likely that the expressed isoforms will be regulated according to tissue type, consistent with existing data [2,4]. Isoforms that are enriched in the brain, and with little to no expression in the cardiovascular system, provide a starting point for the development of brain-selective compounds.

Our findings highlight how incomplete our understanding of the transcriptome remains. This information is crucial, since studies that consider gene expression only at the gene level can miss important switches occurring at the level of individual isoforms. The single nucleotide polymorphisms associated with psychiatric illness within CACNA1C are non-coding, so may act via effects on isoform expression [1]. Understanding the full-length isoform context is also needed to elucidate the interactive effects of splicing variation on protein function, as splicing events that are distant in genomic space may be in close proximity in the mature protein. Notably, the poor understanding of isoform diversity also pertains to model systems, e.g. rodents and stem cell models. Given profound changes in isoform profiles between species and across development $[4,6]$, it is important to characterise isoform profiles in these systems to ensure relevance for pathophysiological studies and drug development. 


\section{Funding and disclosures}

EMT is in receipt of research funding from J\&J Innovations, Biogen and Boehringer Ingelheim, and has provided consultancy for Ono Pharmaceuticals. This research was supported by the National Institute for Health Research Oxford Health Biomedical Research Centre. The views expressed are those of the authors and not necessarily those of the NHS, the NIHR or the Department of Health. The authors have no conflicts of interest to declare in relation to the work described.

\section{Author contributions}

The authors contributed equally to the writing and editing of this document.

\section{References}

1 Harrison PJ, Tunbridge EM, Dolphin AC, Hall J. Voltage-gated calcium channel blockers for psychiatric disorders: genomic reappraisal. The British Journal of Psychiatry. 2020;216(5):250-53.

2 Striessnig J, Pinggera A, Kaur G, Bock G, Tuluc P. L-type Ca2+ channels in heart and brain. Wiley Interdisciplinary Reviews: Membrane Transport and Signaling. 2014;3(2):15-38.

3 Soldatov, N. M., Bouron, A., Reuter, H. Different voltage-dependent inhibition by dihydropyridines of human $\mathrm{Ca} 2+$ channel splice variants. Journal of Biological Chemistry, 1995;270(18): 10540-10543.

$4 \quad$ Mazin PV, Khaitovich P, Cardoso-Moreira M, Kaessmann H. Alternative splicing during mammalian organ development. Nature Genetics. 2021;53(6):925-34.

5 Clark MB, Wrzesinski T, Garcia AB, Hall NAL, Kleinman JE, Hyde T, et al. Long-read sequencing reveals the complex splicing profile of the psychiatric risk gene CACNA1C in human brain. Molecular Psychiatry. 2020;25(1):37-47.

6 Jaffe AE, Straub RE, Shin JH, Tao R, Gao Y, Collado-Torres L, et al. Developmental and genetic regulation of the human cortex transcriptome illuminate schizophrenia pathogenesis. Nature Neuroscience. 2018;21(8):1117-25. 